\title{
ANÁLISIS
}

\section{Acuerdos financieros entre China, Brasil y México: currency swaps}

DOI: $10.32870 /$ mycp.v3i8.463

Gabriela Correa López ${ }^{1}$

\section{Resumen}

La República Popular China (China) tuvo en el año 2013 dos momentos importantes en su relación con Brasil y México. Con Brasil estableció un acuerdo de intercambio de divisas en el marco de la organización de países BRICS, y con México a partir de la visita de Estado del presidente Xi Jinping se anunciaron variados mecanismos de cooperación, con los cuales se espera reasumir la importancia de las relaciones bilaterales.

En este trabajo se analiza el significado de ambos eventos en el contexto de las relaciones internacionales de China con dos países en América Latina con distintos caracteres: una cercana colaboración económica y política en foros internacionales con Brasil, y la recuperación de estilos de colaboración de una relación que se había distanciado en términos económicos y políticos con México.

Palabras clave: China, Brasil, México, cooperación, currency swaps.

Artículo recibido el 08 de septiembre de 2013 y dictaminado el 12 de diciembre de 2013.

1. Profesora-investigadora, UAM-Iztapalapa. Doctora en Relaciones Internacionales por la Universidad de Colima. ORCID http://orcid.org/0000-0001-5725-3496 Correo electrónico: gcl@xanum.uam.mx 


\title{
FINANCIAL ARRANGEMENTS BETWEEN CHINA, BRAZIL AND MÉXICO: CURRENCY SWAPS
}

\begin{abstract}
The People's Republic of China (China) had in 2013 two relevant moments in their relationship with Brazil and Mexico. With Brazil established a currency swap deal within BRICS, and since the State visit of the President Xi Jinping to México have been announced cooperation mechanisms which are expected to resume importance of bilateral relations.

This paper analyzes the significance of both events in the context of China's international relations with two countries in Latin America with different characters: a close economic and political cooperation with Brazil at international forums, and recovery of collaborative styles in economic and political terms with Mexico.

Keywords: China, Brazil, Mexico, cooperation, currency swaps.
\end{abstract}

\section{Acuerdos financieros de cooperación bilateral}

La República Popular China (China) tuvo en el año 2013 dos momentos importantes en su relación bilateral con Brasil y con México. Con Brasil estableció un acuerdo de intercambio de divisas en el marco de la organización de países BRICS, y con México a partir de la visita de Estado del presidente Xi Jinping se anunciaron variados mecanismos de cooperación que reasumen la importancia de las relaciones bilaterales.

En este trabajo se analiza el significado de ambos eventos en el contexto de las relaciones internacionales de China con dos países en América Latina. El tipo de relaciones de China con México y con Brasil es distinto, aunque algunos mecanismos de cooperación e intercambio comercial forman parte de la misma estrategia política de China. El resultado es una cercana colaboración económica y política en foros internacionales con Brasil, y la oferta de recuperación de estilos de colaboración de una relación que se había distanciado en términos económicos y políticos con México.

Entre las reformas previstas pero aún no ejecutadas en China, destacan por su importancia la del sistema bancario y una mayor apertura de la cuenta de capital de la balanza de pagos. En ambos temas se han registrado políticas 
controladas en zonas experimentales para evaluación de nuevas medidas, antes de una extensión a nivel regional o nacional. Cabe destacar las medidas anunciadas por el Banco Central de China en diciembre de 2013 respecto a una lista con mecanismos a implementar en los siguientes tres años relativos a movimientos financieros a través de Hong Kong y las actividades asociadas a la Zona de Libre Comercio de Shanghai.

Esta zona de libre comercio es una de las más importantes experiencias para las intenciones de avanzar en la liberalización financiera y bancaria, de ahí que los 30 puntos dados a conocer como futuras medidas de regulación del Banco Central de China mantienen las expectativas por una mayor apertura financiera. Entre las medidas anunciadas, se aceptará que residentes chinos registrados en la zona mantengan cuentas, en tanto que los extranjeros no residentes podrán obtener cuentas bancarias, así que las transacciones entre estas cuentas y otras fuera de la zona serán consideradas como flujos transfronterizos, considerando cierta flexibilidad para trasladar recursos a cuentas en el exterior. Estas consideraciones no garantizan convertibilidad del yuan a los cuentahabientes, así como tampoco involucran un calendario específico más allá de anunciar que serán adoptadas en el año 2014, como la primera etapa del proyecto de tres años para la Zona de Libre Comercio de Shanghai (China Economic Review, 2013).

La propuesta de impulsar el uso del yuan (renminbi) como

La propuesta de impulsar el uso del yuan (renminbi) como medio de pago de transacciones comerciales, moneda de denominación de inversiones y moneda para resguardar valor de activos, forma parte de las metas del $12^{\circ}$ Plan Quinquenal (20112015). Desde ese año han sido tres los mecanismos impulsados con ese objetivo: el establecimiento de acuerdos bilaterales entre bancos centrales para intercambiar divisas (currency swaps), acuerdos de pagos de comercio bilateral denominados en monedas domésticas y acuerdos de inversiones en instrumentos financieros denominados en yuanes 
medio de pago de transacciones comerciales, moneda de denominación de inversiones y moneda para resguardar valor de activos, forma parte de las metas del $12^{\circ}$ Plan Quinquenal (2011-2015). Desde ese año han sido tres los mecanismos impulsados con ese objetivo: el establecimiento de acuerdos bilaterales entre bancos centrales para intercambiar divisas (currency swaps), acuerdos de pagos de comercio bilateral denominados en monedas domésticas y acuerdos de inversiones en instrumentos financieros denominados en yuanes. Los mecanismos y acuerdos han sido promovidos desde 2009, pero en la región asiática fue 2012 el año de mayor impulso.

Un caso significativo por la relevancia de acuerdos es el de Nigeria, que en agosto de 2012 acordó cambiar 500 millones de dólares de sus reservas internacionales en el banco central a una denominación en yuanes, considerando que China pagaría así con mayor facilidad las importaciones de petróleo que realiza.

Este tipo de acuerdos tienen, además de ventajas al intercambiar monedas domésticas en pagos de comercio, la posibilidad de evitar costos de transacción asociados al cambio a una tercera moneda, generalmente el dólar estadounidense, que es la moneda en la cual se cotizan commodities y petróleo.

Los intereses para establecer este tipo de acuerdos son fuertes si el saldo del comercio bilateral es superavitario para el país asociado con China, ya que además de lograr la diversificación de reservas internacionales, pueden alejarse del dólar y euro en el contexto de inestabilidad internacional y depreciación de estas monedas en los últimos años, ofreciendo alternativas a los pagos de comercio bilateral.

Por ejemplo, para los importadores nigerianos de productos chinos, pagar con yuanes aportados por los exportadores de petróleo a China (o por los inversionistas chinos en Nigeria), les permite alejarse de las fluctuaciones del dólar. El acuerdo currency swaps entre bancos centrales posibilita a bancos comerciales y otros agentes financieros evitar conversiones adicionales entre monedas y operar con una moneda extranjera disponible en el país. Hay que recordar que China mantiene grandes proyectos de exploración y explotación petrolera en Nigeria, así que muchas de sus operaciones comerciales podría hacerlas en yuanes, que serían aceptados con mayor facilidad por bancos comerciales para ser depositados en el banco central. ${ }^{2}$

2. Irán también acepta yuanes por pagos de exportaciones petroleras a China, en tanto que Malasia mantiene yuanes como divisa en sus reservas internacionales. 
Lo relevante detrás de este tipo de acuerdos bilaterales que logra China, además de la expansión de la cooperación financiera y la proyección política de cooperación financiera, es la capacidad que obtiene derivada de su condición superavitaria en términos del comercio bilateral, de trasladar al exterior una posible expansión monetaria. Logra además una mejoría en el costo de administración de sus reservas internacionales, así como el fortalecimiento de su moneda doméstica sin haber liberado totalmente su cuenta de capital, no tener plena convertibilidad del yuan y sin haberse convertido en el principal país acreedor en el mundo.

Técnicamente, los currency swaps son acuerdos para operaciones de arbitraje entre mercados parcialmente segmentados; es decir las divisas que captan los exportadores no se destinan por completo para atender las demandas de los importadores, así que al intervenir los bancos centrales y permitir a los bancos comerciales cambiar mayores volúmenes de moneda extranjera por nacional (o la nacional por extranjera) se mejora la disponibilidad y por tanto los diferenciales entre tipos de cambio tienden a reducirse. El acuerdo currency swap involucra especificaciones sobre rangos de tipos de cambio, tasas de interés, plazos y montos respecto a las tenencias cruzadas de moneda extranjera en el banco central respectivo.

En otras palabras, currency swaps son operaciones normales de intercambio de monedas que realizan los bancos centrales; lo distintivo de los acuerdos promovidos por China es el relativamente alto volumen relacionado con las transacciones comerciales y el plazo de vigencia del acuerdo, que normalmente se renueva en cada vencimiento.

Con el nuevo gobierno que inició en marzo de 2013 presidido por Xi Jinping y en una visita a la región de Qianhai en la provincia de Shenzhen, se reforzó la idea de promover a través del Plan de la Industria Financiera Qianhai la promoción de la internacionalización del yuan como una prioridad entre las innovaciones financieras que se experimentan. En enero de 2013 se inició un esquema de créditos provenientes de bancos en Hong Kong denominados en yuanes, a los que pudieron acceder empresas establecidas en la zona de Qianhai; además de que otros bancos de propiedad estatal fueron autorizados a crear un fondo de inversión en yuanes con recursos de inversionistas registrados en Hong Kong.

Si bien estos dos mecanismos señalados aprovechan la posibilidad de operaciones financieras bajo reglas transfronterizas entre el continente de China y Hong Kong al mismo tiempo que se mantienen distintas regulaciones, resul- 
tan atractivas las posibilidades para los inversionistas, que podrían comprar productos financieros en el mercado cambiario de Qianhai y la nueva Zona de Libre Comercio de Shanghai, en tanto los inversionistas en el continente podrían acceder a compras accionarias de empresas registradas en Hong Kong.

Estos acuerdos que establece China permiten el aumento de la circulación financiera del yuan entre fronteras, sin abrir totalmente la cuenta de capital en el continente chino y, de resultar las experiencias con una evaluación exitosa, confirmaría la decisión del Gobierno chino por encontrar mecanismos de expansión internacional de su moneda. En este experimento China mantiene el tipo de cambio y las tasas de interés controladas, variables que representan sus límites para la apertura de la cuenta de capital, que no está en condiciones de realizar dada la fragilidad de su sistema bancario y la resistencia política de algunos grupos desconfiados de la escasa regulación del mercado financiero mundial.

La experiencia de los países de la región asiática respecto a currency swaps es amplia. Desde el año 2000 se integró la Iniciativa Chiang Mai como red de swaps, anidada en ASEAN + 3, con ampliación de recursos y gestiones que progresan hacia un sistema de fondos autoadministrados. La crisis europea después de 2009 mostró que los fondos de estabilización para liquidez de corto plazo son efectivos, a pesar de que el acceso al tramo principal en la Iniciativa Chiang Mai se mantenga condicionado a un programa económico supervisado por el Fondo Monetario Internacional (FMI).

Tener yuanes en bancos centrales distintos al de China puede ser utilizado para pagar exportaciones de China, lo cual se interpreta como creación de comercio e inversión. Además están presentes en la internacionalización del yuan, motivaciones políticas para brindar reconocimiento a una moneda que técnicamente no cumple con las características para ser considerada divisa fuerte (Takatoshi, 2013: 1).

La red de acuerdos de China que incluyen currency swaps está establecida con 14 economías: Japón, República de Corea (Corea), Mongolia, Ucrania, Nigeria, Kazajstán, Tailandia, Australia, Singapur, Malasia, Indonesia, Brasil y Argentina. En el caso del acuerdo con Taiwán, se considera un acuerdo económico dado que no se reconoce personalidad jurídica a dicho territorio. Otros acuerdos entre bancos centrales respecto a divisas incluyen a Bielorrusia, Islandia, Nueva Zelanda, Uzbekistán, Pakistán, Emiratos Árabes Unidos y Turquía. 
Japón ha disminuido los recursos involucrados en el acuerdo de intercambio de divisas que mantenía con China desde octubre de 2011, pasando de un volumen de 70 mil millones de dólares a sólo 10 mil millones en julio de 2013. Corea dejó sin renovar un acuerdo similar que tenía con Estados Unidos, en tanto aumentó el acuerdo con China a 56 mil millones de dólares con vigencia hasta el año 2017.

Estos movimientos podrían interpretarse como reflejo de una estrategia bajo el nuevo Gobierno de Corea, que estaría buscando alejarse de Estados Unidos y Japón, exponiendo así la importancia de China como su primer socio comercial, y políticamente por las posibilidades de apoyo chino respecto a la futura solución de los problemas con Corea del Norte (Han, 2013: 2).

\section{Dificultades cambiarias en el sistema internacional}

Las primeras recomendaciones del FMI luego de la crisis de 2008 fueron en el sentido de aplicar estímulos fiscales intentando contener la caída de la actividad económica. Luego de rescates y programas de reestructuración severos en algunos países europeos, se presenta un panorama de lenta recuperación y discusiones sobre políticas monetarias expansivas en los países desarrollados que llevan a apreciación del tipo de cambio de países en desarrollo.

Después del año 2012 se ha discutido sobre "guerras cambiarias" y la evidencia de las tendencias de tipos de cambio apunta a confirmar la apreciación resultante, a pesar de la discusión sobre la medida y métodos de cálculo.

En la gráfica 1 se presenta la tendencia de los tipos de cambio nominales de Estados Unidos, China, Brasil y México en términos de derechos especiales de giro (DEG) como una aproximación a la evolución del tipo de cambio de cada moneda. Cabe aclarar que dado que el dólar estadounidense forma parte de las monedas utilizadas para el cálculo del numerario de los DEG, se presenta un sesgo respecto a la evolución del dólar. Lo que destaca en esta gráfica es la alta fluctuación del real brasileño después del año 2009, que a pesar de haberse corregido posteriormente, se mantiene cercano a los niveles del año 2008.

En la misma gráfica 1 se observa una tendencia a la mejoría del yuan, cuya paridad respecto al dólar estadounidense ha pasado de cerca de ocho dólares en 2005 a casi seis yuanes por dólar en enero de 2013. Para México, a pesar de las oscilaciones, el rango de variación el tipo de cambio resulta relativamente estable. 


\section{Gráfica 1}

Tipos de cambio nominales expresados en DEG

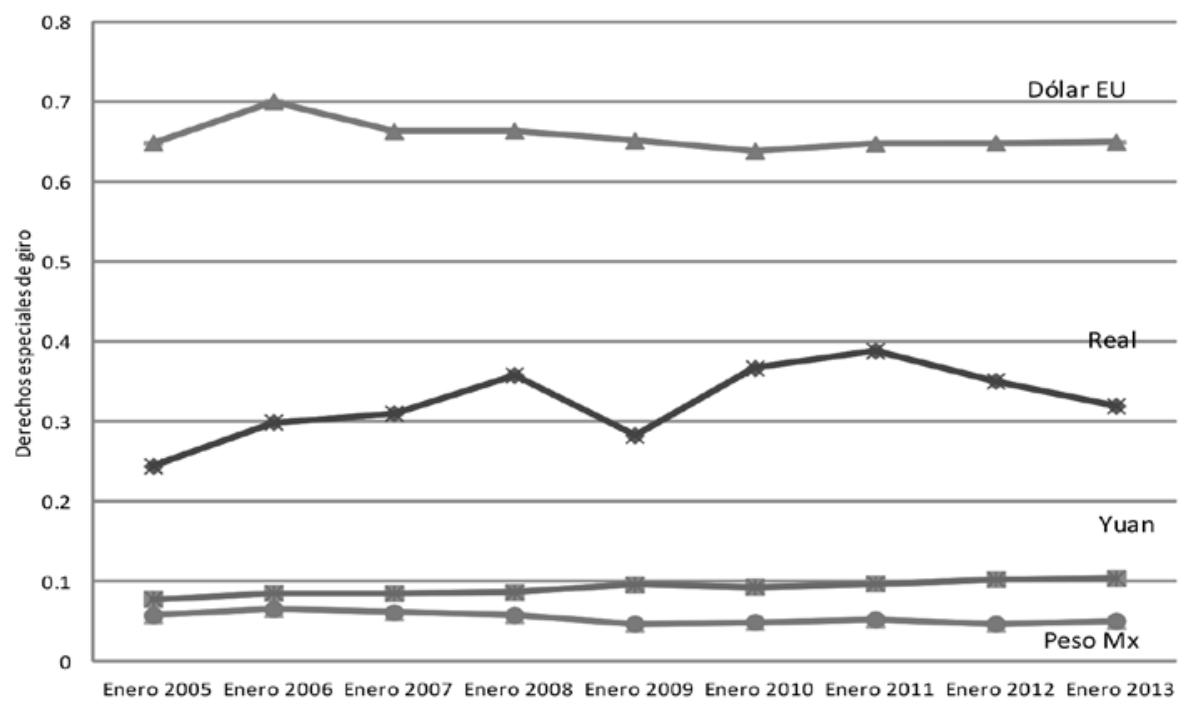

Las fluctuaciones del real brasileño generan dificultades de comercio exterior para Brasil como resultado de los movimientos cambiarios y de entrada de capitales, y son las que han acentuado la posición litigante de Brasil contra las políticas monetarias expansivas de países desarrollados frente a recomendaciones de línea dura para países en desarrollo, así es como ha logrado con sus argumentos e intervenciones que se delimiten temas que deben ser atendidos por el FMI y aquellos que a pesar de ser debatidos en la Organización Mundial de Comercio (OMC) no sean sujetos de interpretaciones que conduzcan a sanciones comerciales.

Estas discusiones han ofrecido oportunidades adicionales de colaboración política de representantes brasileños y chinos en las organizaciones internacionales y esa colaboración ha contribuido a convertir a Roberto Azevedo, durante muchos años representante brasileño en foros internacionales, en director de la OMC a partir de septiembre de 2013.

La gráfica 2 forma parte de un documento técnico de discusiones de un grupo de trabajo de la OMC que por petición de Brasil y otros países ha abordado el debate especializado sobre impactos de política monetaria en diferencias comerciales. La evolución de los datos que se grafican señala la identificada como desalineación del tipo de cambio del real desde el año 2007, 
lo cual ha producido tasas efectivas reales más altas que las que señalarían los indicadores fundamentales del comercio exterior y de los movimientos de capitales en Brasil. Esta evolución es la que Brasil argumenta como dañina a su comercio exterior, resultante de políticas monetarias expansivas en países desarrollados.

\section{Gráfica 2}

Desalineación cambiaria en Brasil
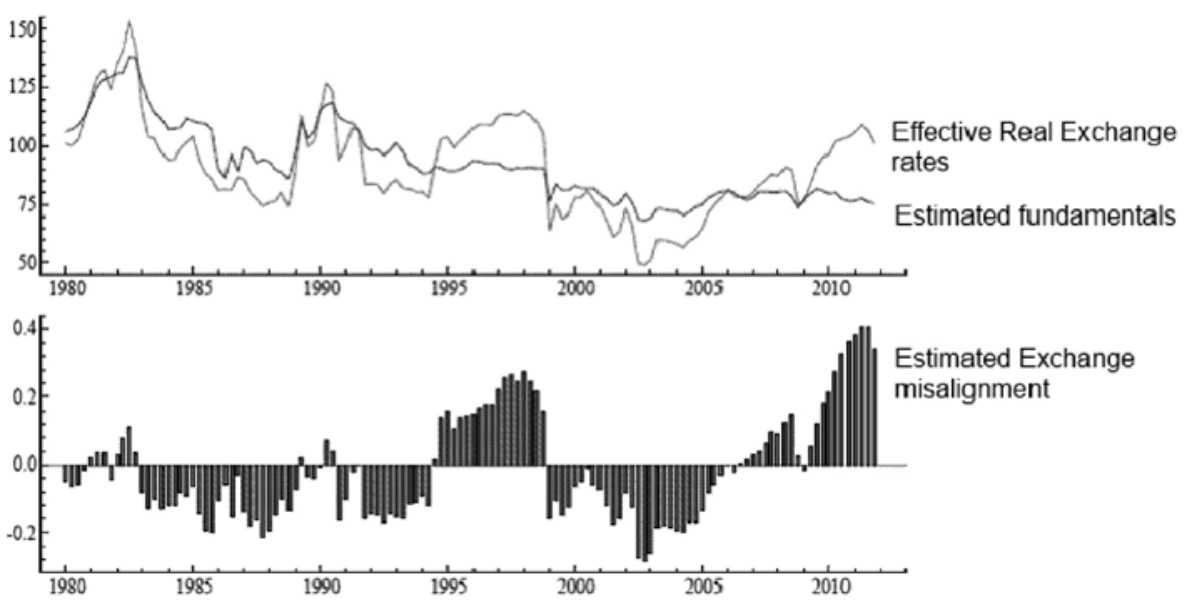

Estimaciones: profesor Emerson Marçal (“Fundação Getúlio Vargas”), julio 2012. Fuente: Tomado de OMC, WT/WGTDF/68.

En el cuadro 1 se presenta la información reportada por el FMI respecto a la evolución de tipos de cambio efectivos reales frente al dólar estadounidense. En estos resultados, que estiman subvaluaciones para el yuan, real y peso mexicano, influyen mecanismos econométricos y de ponderación, por lo cual resulta difícil su interpretación directa. En los resultados de los mecanismos de supervisión aplicados bajo el artículo $4^{\circ}$ del Convenio Constitutivo del FMI y que se publicaron para China en julio de 2013 y Brasil en 2012, se afirma que las desalineaciones no son sustantivas y sobre todo no son resultado de intervenciones directas de los gobiernos. En otras palabras, para el FMI los problemas de tendencias cambiarias no son graves y no son causados por los gobiernos (FMI, 2012, 2013). 


\section{Cuadro 1}

Evolución del tipo de cambio frente al dólar estadounidense

\begin{tabular}{|c|c|c|c|c|c|c|c|}
\hline & 2007 & 2008 & 2009 & 2010 & 2011 & $2012 \mathrm{e}$ & 2013 e \\
\hline \multicolumn{8}{|l|}{ China } \\
\hline Tipo de cambio nominal & 7.604 & 6.945 & 6.831 & 6.77 & 6.461 & 6.3736 & 6.2 \\
\hline TC efectivo real & 4 & 9.2 & 3.4 & -0.5 & 2.7 & 5.7 & -0.2 \\
\hline \multicolumn{8}{|l|}{ Brasil } \\
\hline Tipo de cambio nominal & 1.95 & 1.83 & 2 & 1.76 & 1.67 & 1.95 & 2.15 \\
\hline TC efectivo real & 8.3 & 5.4 & 0.3 & 14.4 & 4.8 & -9 & -9 \\
\hline \multicolumn{8}{|l|}{ México } \\
\hline Tipo de cambio nominal & 10.93 & 11.13 & 13.51 & 12.64 & 12.42 & 13.21 & 12.8 \\
\hline TC efectivo real & 2.9 & 4.5 & 13.4 & -7.9 & 1.5 & 4.7 & -1 \\
\hline
\end{tabular}

Tipo de cambio efectivo real: promedio ponderado considerando los precios de los principales socios comerciales.

Fuente: FMI, IFS, e-library.

Al dibujar estos datos en la gráfica 3, destaca la variabilidad en las estimaciones del FMI de los tipos de cambio efectivos reales, particularmente la del real brasileño. México y China también han enfrentado severas fluctuaciones que, en todo caso, los colocan en estimaciones para 2013 de subvaluación respecto al año 2007.

Gráfica 3

Tipo de cambio efectivo real, var \%

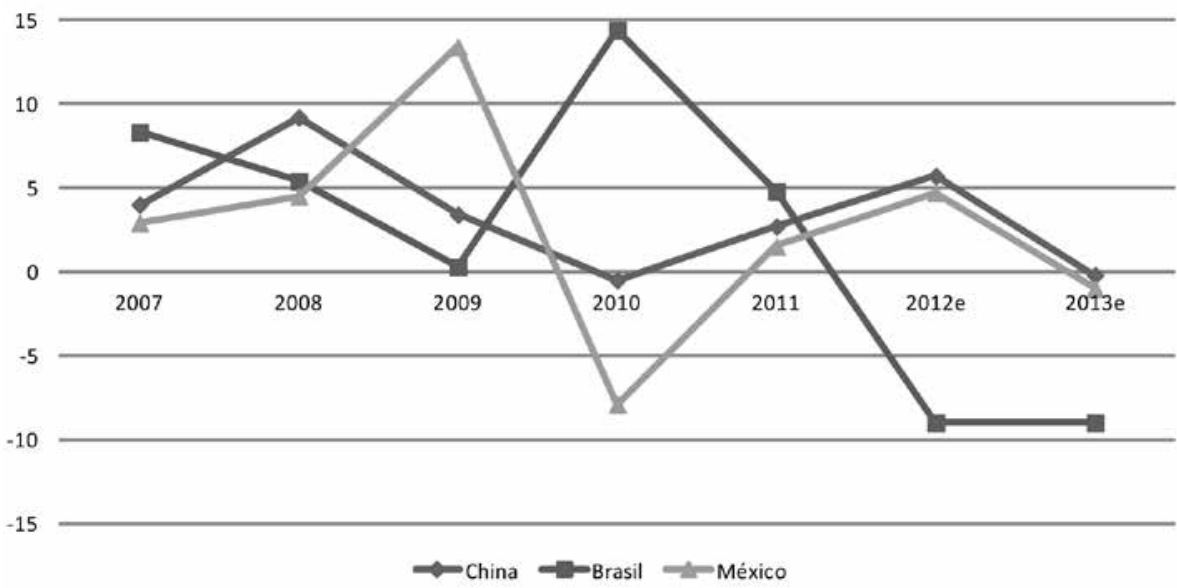


Las contradicciones entre los valores estimados, el sentido de la desalineación y el reconocimiento del FMI de que no corresponden a intervenciones de los gobiernos, intensifican los debates en foros y organizaciones internacionales en donde se han reforzado las posiciones entre planteamientos de funcionarios chinos y brasileños.

Este estilo de colaboración política en materia técnica en variados foros internacionales entre Brasil y China se expresó en abril de 2013 en las reuniones del FMI. Guido Mantega (ministro de Hacienda de Brasil) participó como representante en nombre de su país y de Cabo Verde, República Dominicana, Ecuador, Guyana, Haití, Nicaragua, Panamá, Surinam, Timor Leste, Trinidad y Tobago, que integran el grupo en las reuniones del FMI.

Mantega señaló que los países emergentes enfrentan la abundancia de capital con políticas pragmáticas para evitar desequilibrios, pero que inevitablemente los países en desarrollo están afectados por la recesión en los países avanzados, considerando que la política fiscal expansiva podría ser efectiva para una recuperación porque hay espacios fiscales en los países avanzados, pero no habrá recuperación si todos los países buscan simultáneamente crecer con exportaciones (Mantega, 2013: 16).

El argumento central del debate sobre los efectos de la entrada de flujos de capital (que es también el caso de México) es que obliga a elevar reservas internacionales, disminuir tasas de interés, aceptar apreciación del tipo de cambio o endurecer política fiscal como medidas convencionales pero que no resultan realistas ni democráticas respecto a la mejora de gobernabilidad comprometida para el sistema internacional.

Las críticas de Mantega hacia las reformas del FMI señalan, por ejemplo:

El FMI debería respaldar estas ideas, no sólo tolerarlas, sino debe cumplir con la promoción de coherencia, no pidiendo a países en desarrollo endurecer su política monetaria en tanto la aflojan los países avanzados. La reforma del FMI está en su punto más bajo, las fechas no se cumplen, la posposición se ha vuelto rutina, se ha vencido la fecha para revisión de la cuota, de la reforma y de la forma de cálculo de la cuota. Desde 2011 las reformas se han pospuesto debido a la falta de ratificación del Congreso de Estados Unidos, así como la oposición de los sobrerrepresentados europeos; se juega con la legitimidad y credibilidad del FMI (Mantega, 2013: 34). 
Las argumentaciones técnicas abordan también la fórmula de cálculo de las cuotas en el FMI y las condiciones planteadas a China para responder a su solicitud de incluir el yuan en la canasta de monedas para calcular los DEG:

Usar el PIB como parte central de la fórmula de cuota es correcto, pero hay que considerar el valor agregado en el país en lugar del PIB total, para evitar la múltiple contabilidad, con lo que se penaliza la diversificación productiva y comercial, así como los procesos de integración productiva entre distintos países (Mantega, 2013: 40).

Los funcionarios brasileños (en voz de Roberto Azevedo) habían debatido con apoyo de los representantes chinos (Zhu Hong) en los grupos de trabajo de la OMC respecto a las relaciones entre tipo de cambio y comercio, debate que incluye trabajos de selección de literatura especializada relevante y discusión en seminarios específicos (WTO, 2012). Los debates destacaron que hay dos situaciones en las que el tipo de cambio afecta el comercio: las fluctuaciones y las desalineaciones, señalando que la literatura especializada se ha desplazado desde el análisis del primer tema hacia la segunda discusión. Así que a pesar de las dificultades de estimación en modelos de equilibrio para tipo de cambio real efectivo por el FMI, se aceptó que el tema cabe en las responsabilidades del FMI y la OMC deberá dejar la solución a aquella institución, sin intentar aplicar interpretaciones o recomendaciones, dado que no está en su mandato evitar que se apliquen compensaciones por aranceles específicos que involucren dumping de divisas (WTO, 2012). ${ }^{3}$

En la OMC las consideraciones técnicas resultan insuficientes, ya que omiten otros posibles impactos, por ejemplo el de sobrevaluación de la moneda de países exportadores que eleva el valor de aranceles específicos restringiendo el acceso a mercados de destino. Brasil y China argumentaron que en la OMC deberían proveerse soluciones rápidas, evitando penalizar el acceso a mercados que resultan de esta forma protegidos por medios cambiarios, lo cual no es competencia de la OMC.

Junto a estos debates técnicos y posiciones de apoyo entre Brasily China, aparece la voluntad de cooperación en otras organizaciones, particularmente

3. Uno de los antecedentes del debate es que en el GATT se establecía la posibilidad de aplicar aranceles específicos en casos de pérdida de competitividad por movimientos cambiarios, con la posibilidad de afectar las categorías de productos que fueran dañadas, sin que fueran consideradas medidas de revancha comercial. 
el Grupo BRICs (Brasil, Rusia, India, China y Sudáfrica). En la Quinta Reunión Cumbre de marzo de 2013 en Durban, Sudáfrica, se refrendó la intención de crear un banco para el desarrollo, un acuerdo contingente de reservas y se anunció un acuerdo currency swaps entre China y Brasil equivalente a $30 \mathrm{mil}$ millones de dólares estadounidenses.

\section{Cooperación China, Brasil y México}

Los bancos centrales de China y Brasil anunciaron que el acuerdo currency swaps para el real y el yuan ascendería a 60 mil millones de reales y $190 \mathrm{mil}$ millones de yuanes, con una vigencia de hasta tres años con la posibilidad de renovación, con el objetivo de impulsar el comercio bilateral entre los dos países. La formalización del acuerdo en el marco de la reunión de BRICS refrendó un espíritu de colaboración con un mecanismo conocido por los bancos centrales: currency swaps.

El acuerdo permite insistir en las posiciones que cuestionan al dólar estadounidense y al euro como divisas fuertes, en lo que coinciden Brasil y China. La estrategia del anuncio del acuerdo argumenta los daños al sistema multilateral de comercio y se refiere a la apreciación del real con resultados de aumento de importaciones y disminución de exportaciones, convirtiéndose en expresión de una política de "empobrecer al vecino", asociada a política monetaria expansiva de los países cuyas monedas son importantes en el sistema internacional de pagos. Así que el intercambio directo de monedas a través de un fondo de compensaciones resulta, además de un mecanismo operativo, un anuncio de cooperación bilateral.

Brasil se ha convertido en origen de abastecimientos para China, resultando en 2012 con superávit comercial cercano a 10 mil millones de dólares, que es el mayor del continente latinoamericano. El contraste con México es el déficit bilateral con China por 50 mil millones de dólares. El sentido y volumen de estos resultados comerciales es clave para el diseño de las distintas estrategias de colaboración.

De acuerdo con Rodríguez (2013: 25), las exportaciones de América Latina a China se han concentrado en mineral de hierro, petróleo y derivados, cobre y soya provenientes de Brasil, Venezuela, Chile y Argentina, en tanto China exporta productos manufacturados. En el caso de México y considerando la declaración gubernamental de equilibrar el intercambio comercial, si se aplicara un crecimiento anual de $28 \%$ significa que en 70 años se lograría dicho 
objetivo. Además, $75 \%$ de importaciones desde China son bienes intermedios y la mitad de los productos exportados a China son materias primas.

Correa (2012: 36) sostiene que China "ofrece milagros" de desarrollo a otros países en desarrollo aunque ese gobierno afirme que no exporta modelos económicos, pero al mismo tiempo construye palancas para su capacidad de negociación internacional y afectar a su favor las relaciones de poder mundiales e institucionales. China ha mejorado sus relaciones institucionales con países de América Latina, es socio del Banco Interamericano de Desarrollo desde 2009, contribuye en CEPAL y promueve la importancia de valor que su demanda doméstica representaría para las exportaciones de materias primas.

CePAl (2013: 33 y 35) reconoce que las importaciones desde China son sujetas a numerosas investigaciones anti dumping desde 2008 y que son los productos que desplazan la producción nacional los investigados con mayor frecuencia. Recomienda tomar una perspectiva coordinada de mediano plazo, concentrándose en la cooperación y complementariedad, en lugar de sofisticar el proteccionismo.

Cepal (2012: 31 y 43) también documenta que China mantiene altas tarifas en áreas de interés para exportaciones de América Latina y el Caribe. Los niveles de protección a cereales, azúcar, bebidas y tabaco son sustanciales, en tanto aranceles menores son para minería, productos forestales, químicos e hidrocarburos, por lo cual esta estructura tarifaria contribuye a la concentración de exportaciones primarias de América Latina y el Caribe. Además, si bien la inversión extranjera china se dirige a los países en el continente, del total de recursos 93\% se destina a los paraísos fiscales de Islas Vírgenes e Islas Caimán, $2.1 \%$ a Brasil y 0.3\% a México.

Ésta es la información dura de las acciones de intercambio comercial con China y son las que quedan en segundo plano ante lo que puede calificarse de ansiedad en los gobiernos latinoamericanos, a la espera de que economías externas rescaten a los países de sus dificultades.

En México, a partir de la visita del presidente chino Xi Jinping en junio de 2013, se puso énfasis en un relanzamiento de la relación bilateral, se informó de nuevos mecanismos, áreas de cooperación y estilo de comercio, así como se denominó la relación bilateral como de asociación estratégica integral. Con esto se marca el inicio de un periodo de expectativas y promesas, de identificación de agendas de cooperación y de caracterización de áreas de interés para China, particularmente en materia energética a partir de la reforma constitucional que aceptará inversión privada en esos sectores. 
El contenido básico de las expectativas en esta asociación estratégica integral incluye componentes utilizados por China en sus relaciones de cooperación y acuerdos de negocios con otros países. Lo insólito es que a pesar del déficit bilateral mexicano, se plantea que uno de los objetivos sea buscar el equilibrio en la relación comercial. ${ }^{4}$

El segundo objetivo propuesto fue reconocer áreas de inversiones para capital chino, particularmente en petróleo, exploración marítima y construcción de obras mayores de infraestructura. En conjunto se acepta que México puede constituirse en una plataforma de exportaciones chinas hacia Estados Unidos, Centroamérica y el Caribe, a la vez que amplía sus exportaciones primarias.

Éste es el elemento a destacar. Se trata de recibir preferencias de acceso comercial y de inversiones bajo el Tratado de Libre Comercio de América del Norte, además de encaminar desde territorio mexicano esfuerzos comerciales hacia Centroamérica y el Caribe, donde hay todavía unos pocos países que no mantienen relaciones diplomáticas con China.

Los acuerdos y proyectos dados a conocer tienen amplia variedad: incluyen el establecimiento de oficinas especializadas para asuntos de China y México en el Banco Mexicano de Comercio Exterior en Beijing y en la Secretaría de Economía en México, la formación de un fondo de inversión entre Eximbank de China y Bancomext por 500 millones de dólares, el otorgamiento de un crédito a Pemex para adquisición de embarcaciones y equipos de operaciones fuera de costa a empresas chinas por mil millones de dólares, ${ }^{5}$ se anunció la expansión de opciones de adquisición de tecnología china de ductos para Pemex, se formará un grupo bilateral de alto nivel para análisis de temas estratégicos, así como un grupo de análisis en materia de inversiones, se promoverá el turismo chino, se establecerán centros de estudios México-China en Beijing y en la UNAM en la capital del país, se ampliarán los programas de enseñanza de mandarín y español, se promoverán seminarios para periodistas y empresarios especializados en temas económicos y financieros, se aumentará el número de becas de intercambio académico y estudios sobre China, y México será sede para la Cumbre Empresarial China Latinoamérica en el año 2015 (Peña Nieto, 2013).

4. Lo cual resulta improbable, como se comentó anteriormente.

5. En el año 2009 la empresa privada América Móvil recibió un monto similar de crédito de un banco chino para la adquisición de equipo de telecomunicaciones. 
Un memorándum de entendimiento para cooperación en construcción de infraestructura "de alto impacto" firmado por la Secretaría de Comunicaciones y Transporte considera la promoción de proyectos portuarios, ferroviarios, carreteros y aeroportuarios con participación china, entre los que destacan: tren rápido DF-Toluca, tren México-Querétaro, tren peninsular, metro de Monterrey, tren ligero Guadalajara, servicios de puertos en Altamira, Guaymas, Matamoros y Veracruz. En este memorándum está referido el proyecto del Corredor Transístmico entre Veracruz y Oaxaca, así como el proyecto de instalar una planta enfriadora de gas para exportación en Salina Cruz, Oaxaca.

Por su parte, en un memorándum de entendimiento con la Secretaría de Energía se acordó la formación de un grupo de trabajo conjunto para valorar posibilidades de cooperación en tecnologías limpias, energías renovables y procesos de captura de $\mathrm{CO}_{2}$ (Secretaría de Energía, 2013). No es gratuita la importancia que el comercio de tecnologías y aditamentos solares ha adquirido en China que ha promocionado este sector industrial, participando crecientemente en el mercado internacional correspondiente, lo cual ha conducido a reclamos por dumping de parte de la Unión Europea y Estados Unidos principalmente (oMc, 2013).

Hay que destacar la importancia que en los proyectos de interés para los chinos tiene la actividad energética mexicana, con una adición al convenio de exportación para llegar a 30 mil barriles de petróleo por día, frente al total de exportaciones de 1.1 millones de barriles diarios. La reducción de exportaciones de petróleo a Estados Unidos (frente a la explotación propia de reservas no convencionales de crudo y gas) ha llevado a plantear la ampliación de exportaciones a China e India, que se mantienen como altos demandantes en el mercado internacional.

Entre los gobiernos subnacionales se ha presentado una oleada de visitas oficiales recíprocas y propuestas, donde por ejemplo el Gobierno del Distrito Federal ha expresado su interés en aceptar inversiones y financiamiento para proyectos asociados a turismo, comunicaciones e informática.

Por su parte el presidente chino identificó 300 áreas de cooperación amistosa, mutuamente provechosa, e informó de la aceptación de importación de carne de cerdo y tequila mexicanos que se detuvieron por represalia china a la reunión presidencial mexicana en el gobierno anterior con el Dalai Lama, así como la disposición para concluir las disputas textiles y de vestido (Peña Nieto, 2013). 
En resumen, se han establecido intenciones de colaboración en numerosas categorías y mecanismos que anuncian que la relación bilateral México-China cubrirá actividades que no se habían planteado anteriormente. El avance de los compromisos hacia finales de 2013 reporta la realización de numerosos encuentros oficiales, de negocios y de intercambio de información que incluye asuntos constitucionales, legales, regulaciones sanitarias para medicamentos, medidas fitosanitarias y de tributación.

El trasfondo más importante de esta expansión en la colaboración bilateral es la confianza en que la entrada de capital estatal y privado chino, encaminado principalmente a exportaciones para terceros mercados, así como la inversión privada en la actividad petrolera y el aumento del abastecimiento de petróleo mexicano permitirán mejorar la actividad económica mexicana utilizando el territorio mexicano como plataforma de exportación china.

La amplitud del déficit bilateral mexicano continuará como uno de los desequilibrios más graves resultado de la política comercial y no es acertado suponer que la cooperación bilateral cambiará el sentido del balance.

En el contexto del sistema internacional, México no se plantea (a diferencia de Brasil) colaborar con China en las estrategias de modificación de sistemas de representación internacional, ni tampoco diversificar sus relaciones con otras economías asiáticas que pudieran ofrecer alternativas a la competencia que representa China tanto en el mercado doméstico como en el internacional.

\section{Comentarios finales}

Perseguir intereses propios antes que actuar en forma benevolente, es una perspectiva que debería adoptar cualquier país que se preocupe por su desarrollo económico e inserción internacional.

China lo hace a través de variados mecanismos, incorporando acciones heterodoxas que parten desde su propio sentido de existencia y se expresan, por ejemplo, en declararse socialismo de mercado, o en operaciones ampliadas de mecanismos convencionales de cooperación financiera como los currency swaps. Lograr la internacionalización del yuan a partir de acuerdos bilaterales, particularmente currency swaps, le permite ampliar sus capacidades de comercio y de sistemas de pagos, sin arriesgar su sistema financiero.

Dominar al dólar estadounidense como divisa será difícil para el yuan mientras no tenga libres movimientos en la cuenta de capitales y ofrezca mer- 
cados financieros con alta liquidez a los inversionistas globales. Pero éste no es el objetivo a lograr por encima de la propia estabilidad económica, así que lo que se verá en el futuro es la ampliación de acuerdos y el fortalecimiento de su proyección como un país con el que pueden establecerse arreglos beneficiosos.

Brasil ha coincidido con China en estrategias de negociación que han resultado exitosas y han establecido agendas de discusión en foros internacionales que redundarán en mejoras de su capacidad de gestión e influencia. Brasil continúa con su tradición de erigirse como representante de países en desarrollo como parte de sus aspiraciones internacionales, enfrenta con nuevos tipos de acuerdos algunas de sus tensiones regionales y promueve desde discusiones técnicas hasta mecanismos de posiciones y cooperación en organizaciones internacionales.

El Gobierno en México tendría que revisar con mayor cuidado el significado de la ampliación de áreas de interés y colaboración con China. Cuestionar las visiones del partido en el Gobierno federal anterior y achacarle los problemas con China es insuficiente e irresponsable. Lo importante será construir una relación que coincida con los intereses propios, ya sea exportar petróleo, aceptar inversión privada en producción de energía, desarrollar plataformas de exportación para Estados Unidos y el Caribe y, sobre todo, reconstruir la planta industrial nacional, que significa recuperar empleos perdidos. México debería además considerar la consistencia y alternativas para asociar política comercial y política exterior. Turismo, comercio e inversión van junto a la imagen internacional del país y a su capacidad de proponer asociaciones con otros países para mejorar su capacidad de negociación en el sistema internacional.

Lo increíble para México es lograr revertir el déficit bilateral a partir del aumento de exportaciones a pesar de la prevista ampliación en productos petroleros, así que es la atracción de inversiones el elemento central de la expansión de las relaciones bilaterales con China. No hay registro de coincidencia o colaboración en foros internacionales y los acuerdos de intercambio de divisas entre bancos centrales forman parte de su operación normal.

En resumen, tanto en acuerdos financieros como en cooperación internacional se aprecia que las tácticas y mecanismos que operan China y Brasil son extremadamente diversos respecto de los que manejan China y México. El sustento de las diferencias está expresado en el sentido del saldo comercial bilateral y en la estrategia de inserción internacional. 


\section{Referencias bibliográficas}

Azevedo, Roberto (2012), "The relationship between exchange rates and international trade. Exchange rate misalignments and trade remedies: A conceptual note by Brazil", Working Group on Trade, Debt and Finance, World Trade Organization, WT/WGTDF/W/68.

Banco Central do Brasil (2013), "Banco Central do Brasil e da China estabelecem acordo de swap de moeda”, Banco Central do Brasil, disponible en: www.bcb.gov.br

Cepal (2012), The People's Republic of China and Latin America and the Caribbean. Dialogue and cooperation for the new challenges of the global economy, junio, Santiago de Chile.

China Economic Review (2013), "The Yuan is still stuck in Shanghai's FTz", Banking \& Finance, diciembre 18, disponible en: www.chinaeconomicreview. com/node/65005

Correa López, Gabriela (2012), "China en el Caribe: Estrategia diplomática y de negocios", México y la Cuenca del Pacífico, 15(43), pp. 15-41.

FMI (2012), Brazil, Article IV Report, junio, disponible en: www.fmi.org/Brazil

- - (2013), People's Republic of China, Article IV Report, (mayo, disponible en: www.fmi.org/China

Han, Intaek (2013), “Currency swaps move South Korea closer to China, away from Japan”, East Asia Forum, julio 18, disponible en: www.eastasiadorum. org/2013/07/18

Mantega, Guido (2013), "Statement", 27 ${ }^{\text {th }}$ Meeting International Monetary and Financial Committee, FMI, disponible en: www.imf.org

Mofcom China (2012), "Emerging economics should be given bigger role", Counselor's Office, diciembre 17.

OMC (2013), "Solución de diferencias, DS437", OMC, disponible en: http:// www.wto.org/spanish/tratop_s/dispu_s/cases_s/ds437_s.htm

Peña Nieto, Enrique (2013), "Mensaje a medios de comunicación en el marco de la visita del presidente de la República Popular China", Presidencia de la República, junio 5, disponible en: http://www.presidencia.gob.mx/visitade-estado-del-presidente-de-china-xi-jinping/

Rivero, M. Fernando (2013), "Políticas actuales de guerra (cambiaria). Un esbozo general", Reporte Macroeconómico de México, IV(6), pp. 39-43, disponible en: http://observatorio.azc.uam.mx 
Rodríguez Montoya, José Francisco (2013), “El intercambio comercial entre China y América Latina: Materias primas a cambio de bienes manufacturados", Reporte Macroeconómico de México, IV(6), pp. 21-26, disponible en: http://observatorio.azc.uam.mx

Secretaría de Energía (2013), Memorándum de cooperación energética, junio 5, disponible en: www.presidencia.gob.mx/articulos-prensa/mexico-y-chinafirman-un-memorando-de-entendimiento-sobre-cooperacion-energetica/

Takatoshi, Ito (2013), "Convergent currencies: Challenges to the Asian financial markets”, East Asia Forum, junio 17, disponible en: http://www. eastasiaforum.org/2013/06/17/convergent-currencies-challenges-to-the-asianfinancial-markets/

Wто (2011), The Relationship between Exchange Rates and International Trade: A Review of Economic Literature, Secretariat wTO, WT/WGTDF/w/57.

Zhu, Hong (2012), “Comments on the Brazilian Proposal”, Permanent Mission of China to WTO, MOFCOM China, noviembre 27. 